\title{
Unified description of compressive modulus revealing multiscale mechanics of living cells
}

\author{
Dongshi Guan $\odot,{ }^{1,2, *}$ Yusheng Shen, ${ }^{2}$ Rui Zhang, ${ }^{2}$ Pingbo Huang, ${ }^{3}$ Pik-Yin Lai, ${ }^{4}$ and Penger Tong $\odot^{2, *}$ \\ ${ }^{1}$ State Key Laboratory of Nonlinear Mechanics, Institute of Mechanics, Chinese Academy of Sciences, Beijing 100190, China \\ ${ }^{2}$ Department of Physics, Hong Kong University of Science and Technology, Clear Water Bay, Kowloon, Hong Kong \\ ${ }^{3}$ Division of Life Science, Hong Kong University of Science and Technology, Clear Water Bay, Kowloon, Hong Kong \\ ${ }^{4}$ Department of Physics and Center for Complex Systems, National Central University, Taoyuan City 320, Taiwan
}

(Received 20 July 2021; accepted 17 November 2021; published 9 December 2021)

\begin{abstract}
How to accurately characterize the modulus of living cells at the whole-cell level with a well-defined measurement geometry and precise mathematical modeling of viscoelastic relaxation is an ongoing challenge in biophysics and mechanobiology. Here, we report combined atomic-force-microscopy (AFM) measurements of stress relaxation and indentation force for 10 cell types ranging from epithelial, muscle, and neuronal cells to blood and stem cells, from which we obtain a unified quantitative description of the compressive modulus $E(t)$ of individual living cells. The cell modulus $E(t)$ is found to have an initial exponential decay at short times $t$ followed by a long-time power-law decay together with a persistent modulus. The three components of $E(t)$ at different timescales thus provide a digital spectrum of mechanical readouts that are closely linked to the hierarchical structure and active stress of living cells. This work provides a reliable experimental framework that can be utilized to characterize the mechanical state of living cells and investigate their physiological functions and diseased states.
\end{abstract}

DOI: 10.1103/PhysRevResearch.3.043166

\section{INTRODUCTION}

A hallmark of active biological materials, such as living cells and tissues, is their emergent mechanical properties exhibiting a mixture of fluid- and solidlike behaviors across multiple spatial and temporal scales [1-6]. Living cells regulate their mechanical properties dynamically together with their shape and volume in a variety of cellular functions and activities [7,8]. For example, mammalian cells become nearly spherical in shape and mechanically stiffer during the mitotic phase of cell division [9-11]. Abnormal changes in the mechanical properties of cells are closely connected to various human diseases and aging [12-15]. For these reasons, the experimental and theoretical efforts aimed at understanding the mechanics of living cells and its relation to the underlying cellular structure and response have recently witnessed a continuing growth.

Various experimental techniques have been recently developed to measure the viscoelastic properties of living cells. Examples include micropipette aspiration [16,17], microplate or colloidal probe indentation [18,19], magnetic and optical tweezers [20,21], and microrheology methods based on single- or two-particle tracking [22,23]. Among these techniques, atomic force microscopy (AFM) has served as an effective and versatile platform for studying a range of prob-

\footnotetext{
*dsguan@imech.ac.cn, penger@ust.hk

Published by the American Physical Society under the terms of the Creative Commons Attribution 4.0 International license. Further distribution of this work must maintain attribution to the author $(s)$ and the published article's title, journal citation, and DOI.
}

lems in mechanobiology, such as cell adhesion, locomotion, differentiation, division, cancerization, cytoskeleton remodeling, and tissue development [9,14,15,24-31].

Although considerable progress has been achieved in recent years, our fundamental understanding of the multiscale mechanics of living cells is often challenged by incomplete experimental characterization and oversimplified theoretical modeling. For example, the Young's modulus $E$ of living cells obtained from different specimens and using experimental techniques under different sampling conditions and at different temporal and spatial scales varies by three orders of magnitude (0.1-100 kPa) [25]. The theoretical models used for calculating $E$ are often oversimplified with assumptions that can hardly accommodate the actual measurement geometry and complex material parameters of living cells. At present, a unified theory that can explain the viscoelastic properties of living cells across different scales remains lacking. The contact mechanics models used in AFM indentation measurements, such as the Hertz, JKR, and DMT models $[27,32,33]$, assume that the living cell is purely elastic and has a constant $E$. Meanwhile, recent stress relaxation measurements show that living cells have a time-dependent relaxation modulus $E(t)$ [4,32,34-37]. A unified description of stress relaxation and indentation measurements is needed in order to understand the viscoelastic nature of living cells across different scales. This description will improve our understanding of the major roles played by the mechanical properties of cells and their response to external mechanical cues in regulating the essential cellular events and functions at an integrated cellular level.

In this paper, we report a systematic study of stress relaxation and indentation force of individual living cells by using an AFM-based force apparatus. With the help of a specially 
(a)



(b)

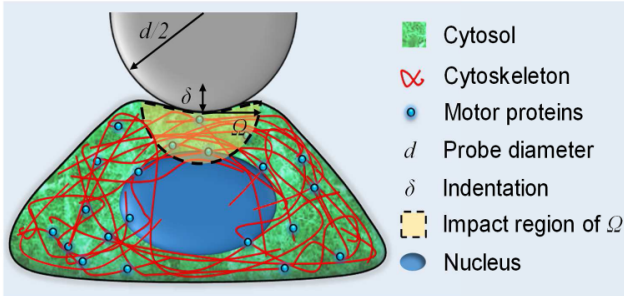



(f)

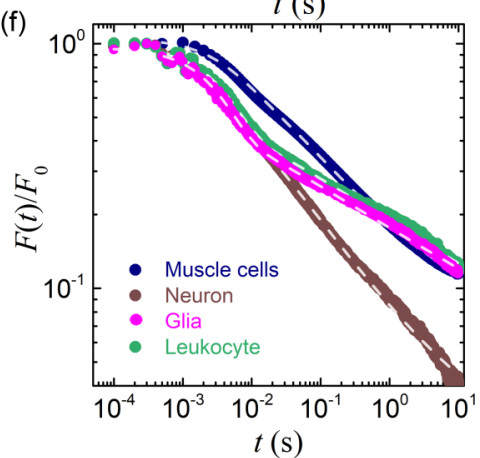

(d)

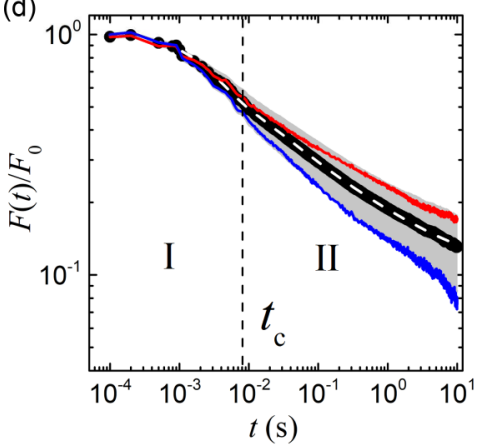

(e)

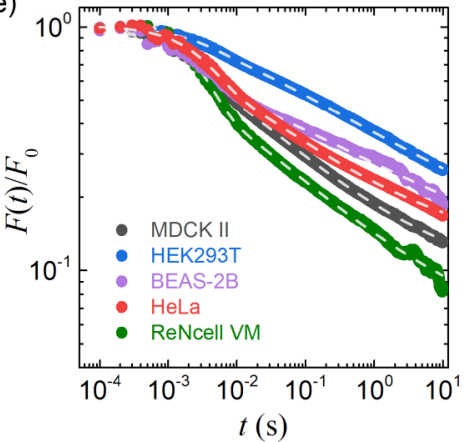

FIG. 1. Cellular stress relaxation reveals a crossover from a short-time exponential decay to a long-time power-law decay. (a) Sketch of force relaxation measurement by AFM for a living cell. Force relaxation $F(t)$ is measured as a function of time $t$ after a constant indentation $\delta$ is quickly applied to the cell at $t \simeq 0$, as shown in the inset of (c). (b) A sketch of the essential mechanical structures inside a living cell responsible for the compressive modulus $E(t)$. When an AFM colloidal probe of diameter $d$ presses the cell surface and generates a downward indentation $\delta$, the impact region in the cell has a radius $\Omega \simeq \sqrt{d \delta / 2}$. (c) Example of the measured $F(t)$ for a living MDCK II cell. The black curve shows a sharp increase of $F(t)$ to reach a preset maximum value $F_{0}=10 \mathrm{nN}$ within $10 \mathrm{~ms}$, as the AFM probe compresses the cell at a constant loading speed $v=100 \mu \mathrm{m} / \mathrm{s}$. The red curve shows the force relaxation when the indentation $\delta(\sim 1 \mu \mathrm{m})$ is held constant. The green dashed line indicates the initial exponential decay of the measured $F(t)$, and the blue dashed line shows the long-time power-law decay to a constant value. (d) Log-log plots of the normalized force relaxation $F(t) / F_{0}$ for MDCK II cells. The black curve is the averaged $F(t) / F_{0}$ over $N=39$ MDCK II cells, and the grey shaded area indicates the standard deviation of the measurements. The red and glue curves show two typical curves of $F(t) / F_{0}$ located near the upper and lower bounds of the standard deviation. The vertical black dashed line indicates the crossover time $t_{c}(\simeq 8.4 \mathrm{~ms})$ from the exponential (I) to power-law (II) decays. (e) Measured $F(t) / F_{0}$ for living cells from five different cell lines. (f) Measured $F(t) / F_{0}$ for four different types of primary cells. The white dashed lines in (d)-(f) indicate the fit of Eq. (1) to the data points, and the fitting results are given in Table I.

designed colloidal probe, we are able to obtain a large volume of simultaneously measured relaxation and indentation curves over a five-decade time span ranging from $0.1 \mathrm{~ms}$ to $10 \mathrm{~s}$ and a two-decade velocity span ranging from 1 to $100 \mu \mathrm{m} / \mathrm{s}$. A central finding of this investigation is that the measured relaxation and indentation curves for 10 different cell types can all be described by a universal compressive modulus $E(t)$, which contains three relaxation modes at different timescales. The modulus $E(t)$ is found to be closely related to the hierarchical structure and active stress of living cells. Our experiment thus provides new insights into the origin of the multiscale mechanics of living cells.

\section{RESULTS}

\section{A. Stress relaxation of living cells reveals a time-dependent compressive modulus $E(t)$}

Force relaxation measurement for a living cell is performed by using an AFM-based force apparatus, as sketched in Fig. 1(a). The AFM probe consists of a glass sphere of diameter $d(7-18 \mu \mathrm{m})$, which is glued on the front end of a rectangular cantilever beam. The size of the glass sphere is chosen to be comparable with the cell size $(10-50 \mu \mathrm{m})$. The glass sphere is coated with a thin layer of poly(L-lysine)-graftpoly(ethylene glycol) to effectively reduce the cell adhesion to the probe [29]. In the experiment, a constant indentation $\delta$ is quickly applied to the cell at a loading speed $v=100 \mu \mathrm{m} / \mathrm{s}$. This loading speed is chosen to be as fast as possible while avoiding unwanted hydrodynamic effects. The value of $\delta$ is typically set at $10 \%-20 \%$ of the cell height $H(5-15 \mu \mathrm{m})$, which gives rise to an impact range of radius $\Omega \simeq \sqrt{d \delta / 2}$ [38], as shown in Fig. 1(b). For the largest probe size $d \simeq$ $18 \mu \mathrm{m}$ and indentation $\delta \simeq 1 \mu \mathrm{m}$ used on a living cell with a height $H \simeq 10 \mu \mathrm{m}$ (such as MDCK II), we have $\Omega \simeq 3 \mu \mathrm{m}$. After the elastic restoring force of the cell reaches its preset maximal value $F_{0}(2-10 \mathrm{nN})$, the AFM records the force relaxation $F(t)$ while keeping the indentation $\delta$ constant, as shown in Fig. 1(c). We are able to measure $F(t)$ over a five-decade time span ranging from $0.1 \mathrm{~ms}$ to $10 \mathrm{~s}$. The long-time limit is set to avoid possible influences from the cytoskeleton remodeling and water penetration through cell membrane $[39,40]$.

Figure $1(\mathrm{~d})$ shows the normalized force relaxation $F(t) / F_{0}$ from 39 MDCK II cells. While the measured $F(t) / F_{0}$ shows some cell-to-cell variations, all the single-cell relaxation curves exhibit two common features. First, the measured $F(t)$ has a fast exponential-like decay at short times (regime I) 
TABLE I. Measured mechanical properties of ten different cell types and their responses to drug treatments. The values of the weighting factor $C_{i}$ for each relaxation mode, the relaxation time $\tau_{1}\left(\simeq \tau_{2}\right)$, and the power-law exponent $\alpha$ are obtained from the averaged force relaxation curves as illustrated in Fig. 1. The absolute values of the modulus $E_{i}$ for each relaxation mode are obtained from the indentation force measurements as illustrated in Fig. 2. In this case, the values of $C_{i}, \tau_{1}, \alpha$, and $E_{i}$ are obtained from the measurements on the same individual cell. The error bars of $E_{i}$ indicate the standard deviations for the number $N$ cells studied in each cell type. Typical error bars of $\tau_{1}$ and $\alpha$ are indicated in Figs. 4(e) and 4(g).

\begin{tabular}{|c|c|c|c|c|c|c|c|c|c|c|}
\hline Cell type & $C_{1}$ & $C_{2}$ & $C_{\infty}$ & $\tau_{1}, \tau_{2}(\mathrm{~ms})$ & $\alpha$ & $\boldsymbol{E}_{\mathbf{0}}(\mathrm{Pa})$ & $\boldsymbol{E}_{1}(\mathrm{~Pa})$ & $\boldsymbol{E}_{\mathbf{2}}(\mathrm{Pa})$ & $\boldsymbol{E}_{\infty}(\mathrm{Pa})$ & $N$ \\
\hline MDCK II [black in Fig. 1(d)] & 0.28 & 0.64 & 0.08 & 2.78 & 0.30 & $2543 \pm 1099$ & $678 \pm 298$ & $1703 \pm 614$ & $162 \pm 73$ & 39 \\
\hline MDCK II [red in Fig. 1(d)] & 0.27 & 0.62 & 0.11 & 2.88 & 0.28 & 2048 & 558 & 1264 & 226 & 1 \\
\hline MDCK II [blue in Fig. 1(d)] & 0.32 & 0.63 & 0.05 & 2.61 & 0.34 & 2378 & 762 & 1502 & 113 & 1 \\
\hline MDCK II (+dATP) & 0.21 & 0.78 & $0.01 \downarrow$ & 3.11 & 0.30 & $2199 \pm 826$ & $371 \pm 103$ & $1813 \pm 590$ & $15 \pm 10$ & 20 \\
\hline MDCK II (+Bleb) & 0.33 & 0.64 & $0.02 \downarrow$ & 2.60 & 0.29 & $1591 \pm 901$ & $358 \pm 136$ & $1210 \pm 470$ & $23 \pm 14$ & 21 \\
\hline MDCK II (+LtnA) & 0.36 & 0.63 & $0.01 \downarrow$ & 3.00 & $0.41 \uparrow$ & $767 \pm 164$ & $250 \pm 82$ & $508 \pm 116$ & $9 \pm 6$ & 20 \\
\hline MDCK II (PFA-fixed) & 0.28 & 0.72 & $\sim 0 \downarrow$ & 6.80 & $0.05 \downarrow$ & $14553 \pm 5389$ & $4165 \pm 1882$ & $10353 \pm 3695$ & $\sim 0$ & 15 \\
\hline HeLa & 0.34 & 0.56 & 0.10 & 3.84 & 0.27 & $3222 \pm 1322$ & $1112 \pm 542$ & $1853 \pm 623$ & $257 \pm 144$ & 33 \\
\hline HeLa (+dATP) & 0.29 & 0.68 & $0.02 \downarrow$ & 4.34 & 0.26 & $2202 \pm 762$ & $352 \pm 175$ & $1827 \pm 569$ & $24 \pm 18$ & 18 \\
\hline $\mathrm{HeLa}(+\mathrm{Bleb})$ & 0.29 & 0.70 & $0.01 \downarrow$ & 4.30 & 0.26 & $1501 \pm 399$ & $357 \pm 158$ & $1111 \pm 353$ & $34 \pm 24$ & 19 \\
\hline $\mathrm{HeLa}(+\operatorname{Ltn} \mathrm{A})$ & 0.23 & 0.77 & $0.01 \downarrow$ & 2.91 & $0.41 \uparrow$ & $952 \pm 145$ & $264 \pm 65$ & $675 \pm 68$ & $14 \pm 11$ & 20 \\
\hline HEK293T & 11 & 0.8 & 0.04 & & 17 & $1228 \pm 475$ & $134+8$ & $1060 \pm 433$ & & 23 \\
\hline BEAS-2B & 0.36 & 0.59 & 0.04 & 3.68 & 0.16 & $2264 \pm 998$ & $703 \pm 396$ & $1461 \pm 576$ & $100 \pm 79$ & 19 \\
\hline ReNcell VM & 0.49 & 0.47 & 0.04 & 2.77 & 0.28 & $3439 \pm 1042$ & $1716 \pm 701$ & $1601 \pm 357$ & $122 \pm 90$ & 16 \\
\hline Muscle cells & 0.12 & 0.82 & 0.06 & 5.08 & 0.37 & $4254 \pm 2258$ & $593 \pm 403$ & $3436 \pm 1376$ & $225 \pm 77$ & 16 \\
\hline Glia & 0.51 & 0.46 & 0.02 & 3.69 & 0.18 & $4083 \pm 1557$ & $2071 \pm 1089$ & $1880 \pm 710$ & $132 \pm 94$ & 19 \\
\hline Neuron & 0.25 & 0.73 & 0.02 & 3.01 & 0.41 & $2025 \pm 708$ & $527 \pm 264$ & $1463 \pm 495$ & $34 \pm 28$ & 20 \\
\hline Leukocyte & 0.48 & 0.48 & 0.04 & 4.50 & 0.18 & $1172 \pm 422$ & $529 \pm 251$ & $580 \pm 200$ & $63 \pm 59$ & 16 \\
\hline Erythrocyte & 0.96 & $\sim 0$ & 0.04 & 2.08 & - & $1723 \pm 483$ & $1657 \pm 471$ & $\sim 0$ & $66 \pm 48$ & 19 \\
\hline
\end{tabular}

followed by a slow power-law decay at long times (regime II). The crossover time $t_{c}$ between these two regimes ranges in several milliseconds. Second, the measured $F(t)$ relaxes to a nonzero value, which is $(14 \pm 6) \%$ of its initial value $F_{0}$ at the longest time $t=10 \mathrm{~s}$. These two common features are also observed in the measured $F(t)$ for other cell types, as shown in Figs. 1(e) and 1(f).

It is found that the measured $F(t) / F_{0}$ for different cell types can all be well described by a common function,

$$
F(t) / F_{0}=C_{1} e^{-t / \tau_{1}}+C_{2}\left(1+t / \tau_{2}\right)^{-\alpha}+C_{\infty},
$$

where $C_{i}$ is the weighting factor of each relaxation mode, $\tau_{1}$ and $\tau_{2}$ are the corresponding relaxation times, and $\alpha$ is the power-law exponent. The last term $C_{\infty}\left(=1-C_{1}-C_{2}\right)$ represents the residual constant force (or stress) in a living cell, which is extrapolated at large $t(\rightarrow \infty)$. The white dashed line in Fig. 1(d) shows the best fit of Eq. (1) to the data with five fitting parameters. The fitted results are given in Table I. It is found that the fitted value of $\tau_{1}(=2.8 \mathrm{~ms})$ is always very close to $\tau_{2}(2.5 \mathrm{~ms})$, and in the following, we set $\tau_{1}=\tau_{2}$ to reduce the number of fitting parameters.

Equation (1) thus quantitatively describes the three relaxation modes of living cells with four fitting parameters. At the crossover time $t_{c} \simeq 3 \tau_{1}(=8.4 \mathrm{~ms})$, we find the ratio of the exponential term to the power-law term in Eq. (1) is about 0.03 , indicating that the exponential decay almost vanishes while the power-law decay becomes dominant for $t>t_{c}$. The constant term $C_{\infty}(\simeq 0.08)$ is important for living cells and cannot be simply ignored (see more discussions below). The measured $F(t) / F_{0}$ for other cell types can also be well described by Eq. (1) [see white dashed lines in Figs. 1(e) and 1(f)], and the fitting results are given in Table I.
With the linear relation $F(t) \sim \varepsilon E(t)$ for a constant strain $\varepsilon=\delta / H$, we find that the time-dependent Young's modulus under compression (namely, compressive modulus) $E(t)$ of living cells can be expressed as

$$
E(t)=E_{1} e^{-t / \tau_{1}}+E_{2}\left(1+t / \tau_{2}\right)^{-\alpha}+E_{\infty},
$$

where $E_{1}, E_{2}$, and $E_{\infty}$ are the elastic coefficients of the three relaxation modes. With the initial modulus $E_{0}$ defined as $E_{0}=$ $E(t=0)=E_{1}+E_{2}+E_{\infty}$, we have $E_{i}=C_{i} E_{0}(i=1,2, \infty)$, where $C_{i}$ is given in Eq. (1).

\section{B. Speed dependence and hysteresis of indentation force $F(\delta)$ results from $E(t)$}

Another mechanical measurement for living cells is the indentation force $F(\delta)$ measured as a function of indentation $\delta=v t$, where $v$ is the loading speed when the AFM probe moves downward against the cell surface (approach) or moves upward away from the cell surface (retract), as shown in Fig. 2(a). The contact point $(\delta=0)$ between the AFM probe and cell surface is defined as the point at which the slope of $F(\delta)$ begins to increase above the noise level. In the experiment, the approaching curve of $F(\delta)$ is measured first until reaching a preset maximal value $F_{0}$, and the AFM probe retracts away from the surface. The value of $F_{0}(2-10 \mathrm{nN})$ is chosen so that the resulting maximal strain $\varepsilon=\delta / H \simeq 0.2$, which is the same as that for the stress relaxation measurement on the same cell. Figure 2(b) shows the measured $F(\delta)$ for a MDCK II cell at three different speeds. The measured approaching (solid lines) and retracting (dashed lines) curves of $F(\delta)$ demonstrate speed dependence and hysteresis. The 
(a)
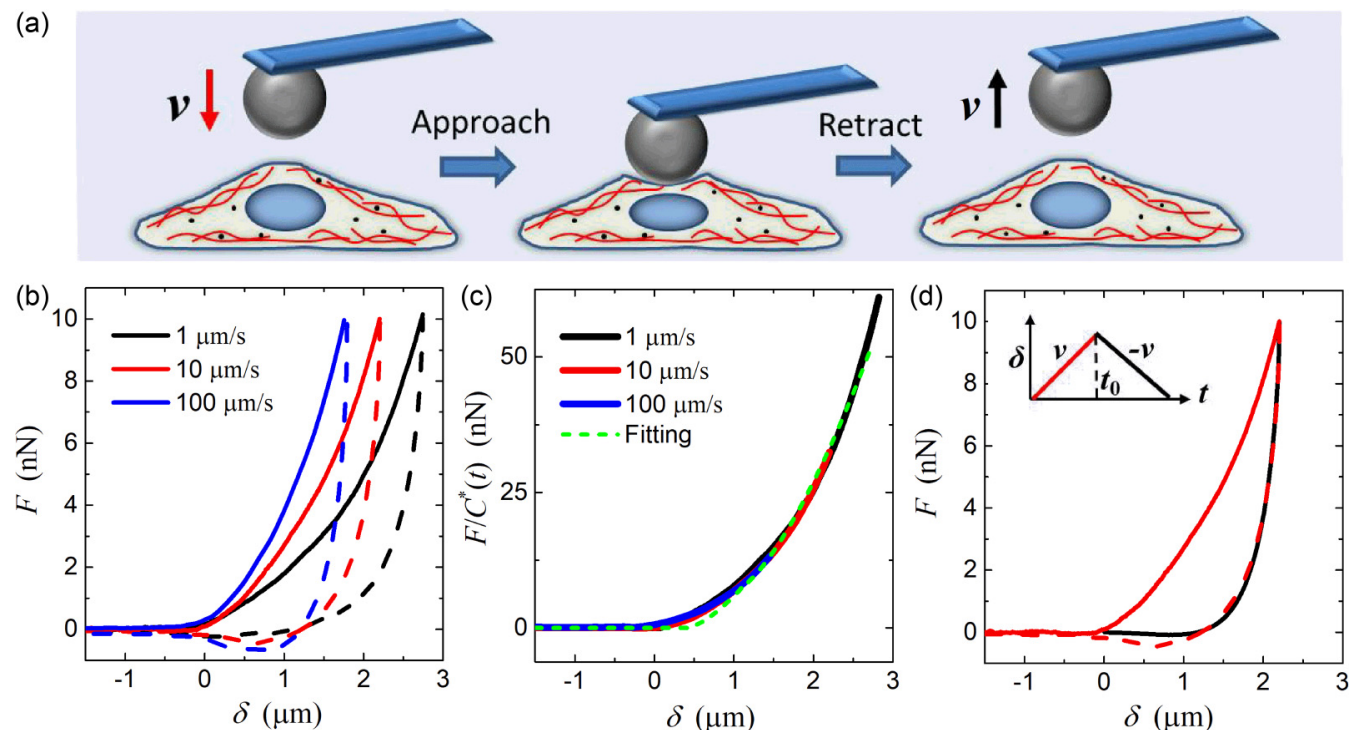

FIG. 2. Cellular indentation force reveals a speed dependence and hysteresis. (a) Sketch of indentation force measurement by AFM for a living cell. The indentation force $F(\delta)$ is measured as a function of indentation $\delta$ when the AFM probe first moves downward against the cell surface at a constant loading speed $v$ (approach) and then moves backward away from the cell surface at the same speed (retract). (b) Measured $F(\delta)$ for a living MDCK II cell at three different speeds: $v=1$ (black curve), 10 (red curve), and $100 \mu \mathrm{m} / \mathrm{s}$ (blue curve). The solid lines are obtained in the approaching direction and the dashed lines are obtained in the retracting direction. (c) Normalized plot of $F(\delta) / C^{*}(t)$ using the three approaching curves shown in (b). The green dashed line indicates the fit of Eq. (4) to the master curve with $E_{0}=3583$ Pa. (d) Replot of the measured $F(\delta)$ at $v=10 \mu \mathrm{m} / \mathrm{s}$ (red curve). The black solid line shows the calculated retracting curve using Eq. (3) with a continuous piecewise linear loading function of $\delta(t)$, as shown in the inset.

cell appears to be stiffer with faster pushing, given that a larger force is required to deform the cell with the same $\delta$.

For a pure elastic body without surface adhesion, the measured $F(\delta)$ is expected to follow the Hertz equation [41], $F(\delta)=\left(4 R^{1 / 2} \delta^{3 / 2} E\right) /\left[3\left(1-v^{2}\right)\right]$, where $R=1 /(2 / d+$ $\left.1 / R_{2}\right)$ is the effective radius at contact with $R_{2}$ being the radius of curvature of the elastic body, $v$ is the Poisson's ratio ( $v \simeq 0.5$ for living cells [19]), and $E$ is the Young's modulus. The Hertz equation predicts that $F(\delta)$ is a single-valued function of $\delta$ and should not exhibit any speed dependence or hysteresis. With the time-dependent modulus $E(t)$ in Eq. (2), we have the modified Hertz equation $[36,42,43]$,

$$
F(\delta, t)=\frac{4 R^{\frac{1}{2}}}{3\left(1-v^{2}\right)} \int_{0}^{t} E\left(t-t^{\prime}\right) \frac{\partial \delta^{\frac{3}{2}}}{\partial t^{\prime}} d t^{\prime} .
$$

With a linear loading $\delta=v t$ for the approaching curve, we obtain

$$
F(\delta, t)=\frac{4 R^{\frac{1}{2}} \delta^{\frac{3}{2}} E_{0}}{3\left(1-v^{2}\right)} C^{*}(t),
$$

where $t=\delta / v$ and

$$
\begin{aligned}
C^{*}(t) \simeq & C_{1} \frac{3}{2}\left\{\frac{\tau_{1}}{t}-\frac{\sqrt{\pi}}{2}\left(\frac{\tau_{1}}{t}\right)^{\frac{3}{2}} e^{-t / \tau_{1}} \operatorname{Erfi}\left[\left(\frac{t}{\tau_{1}}\right)^{1 / 2}\right]\right\} \\
& +C_{2} \frac{3 \sqrt{\pi} \Gamma(1-\alpha)}{4 \Gamma\left(\frac{5}{2}-\alpha\right)}\left(\frac{t}{\tau_{2}}\right)^{-\alpha}+C_{\infty} .
\end{aligned}
$$

In the above, $\operatorname{Erfi}[x]$ is the imaginary error function and $\Gamma(x)$ is the gamma function (see Appendix A 1 for more details). Equation (4) takes the Hertz form, once $F(\delta, t)$ is normalized by $C^{*}(t)$.
Figure 2(c) shows a normalized plot of $F(\delta, t) / C^{*}(t)$ using the same approaching data shown in Fig. 2(b). Indeed, the three approaching curves obtained at different speeds collapse into a single master curve, which is well described by Eq. (4) (green dashed line) with a single fitting parameter $E_{0}=3583 \mathrm{~Pa}$. By using $E_{i}=C_{i} E_{0}$, we find $E_{1}=1003 \mathrm{~Pa}$, $E_{2}=2293 \mathrm{~Pa}$, and $E_{\infty}=287 \mathrm{~Pa}$. With the measured $E(t)$, we can also calculate the retracting curve by using Eq. (3) with a continuous piecewise linear loading function $\delta(t)$, as shown in the inset of Fig. 2(d) (see Appendix A 3 for more details). As shown in Fig. 2(d), the calculated retracting curve agrees well with the data, except for a slight deviation at small $\delta(<1 \mu \mathrm{m})$. This deviation is likely caused by a weak adhesion between the AFM probe and cell surface.

Figure 2 thus demonstrates that the observed speed dependence and hysteresis in the measured $F(\delta)$ are caused by the time-dependent compressive modulus $E(t)$ and that our multiscale modeling of $E(t)$ in Eq. (2) captures the essential physics. The two sets of AFM experiments complement each other, with the force (or stress) relaxation measurement determining the functional form of $E(t)$ and the indentation force measurement further determining the absolute value of $E(t)$ and validating the stress relaxation measurement. This unified description of multiscale cell mechanics is found to be applicable to ten different cell types studied and their mechanical properties are summarized in Table I. In the experiment, we measure $F(\delta)$ and $E(t)$ simultaneously on the same cell, and calculate $C^{*}(t)$ with Eq. (5) by using the fitting parameters from the measured $E(t)$ of the same individual cell. In this way, we obtain consistent results with reduced systematic errors and the error bars shown in Table I result mainly from cell-to-cell variations. 



FIG. 3. Speed dependence of cellular stress relaxation. (a) Measured $F(t) / F_{0}$ for a MDCK II cell at different loading speeds: $v=1$ (blue), 10 (red), and $100 \mu \mathrm{m} / \mathrm{s}$ (black). The yellow solid lines show the exponential fits to the data points at short times. The green dashed lines show the calculated $F(t) / F_{0}$ using Eq. (3) with a continuous piecewise linear loading function of $\delta(t)$, as shown in the inset. (b) Obtained apparent exponential decay time $\tau_{1}^{\prime}$ as a function of $v$. (c) Measured $F(t) / F_{0}$ for a MDCK II cell at $v=100 \mu \mathrm{m} / \mathrm{s}$. The measurements are made with different maximal forces (or indentations): $F_{0}=10$ ( $\delta=1.6 \mu \mathrm{m}$, black), $20(\delta=2.2 \mu \mathrm{m}$, red), and $30 \mathrm{nN}(\delta=2.6 \mu \mathrm{m}$, blue).

\section{Characteristics of exponential decay at short times}

To understand the physical origin of the exponential decay in $E(t)$, we conduct force relaxation measurements with varying loading speeds $v$ and maximal forces $F_{0}$. As shown in Fig. 3(a), the initial decay of the measured $F(t) / F_{0}$ becomes slower with decreasing speed $v$. As a result, the crossover time to the long-time power-law decay is delayed up to $0.3 \mathrm{~s}$ for the slowest loading speed $v=1 \mu \mathrm{m} / \mathrm{s}$. It is found that the initial decay of the measured $F(t) / F_{0}$ can still be fit to a simple exponential function (yellow solid lines), but the obtained apparent decay time $\tau_{1}^{\prime}$ changes with $v$. As shown in Fig. 3(b), the obtained $\tau_{1}^{\prime}$ decreases with increasing $v$ and situates at a stable value $\tau_{1}$ when $v \gtrsim 100 \mu \mathrm{m} / \mathrm{s}$. Therefore, we choose $v=100 \mu \mathrm{m} / \mathrm{s}$ to conduct all the stress relaxation measurements, so that the obtained $\tau_{1}$ is an intrinsic decay time independent of $v$.

As shown in Fig. 3(c), the obtained $F(t) / F_{0}$ and $\tau_{1}$ are not sensitive to the magnitude of the maximal force $F_{0}$ or indentation $\delta$ used in the measurement. We also find that the measured $F(\delta)$ follows the modified Hertz equation (4) when the maximal strain $\varepsilon=\delta / H \lesssim 0.3$. These results indicate that the mechanical response of the AFM probed region is linear and is not influenced very much by the nucleus and substrate, whose mechanical responses should be significantly different. Furthermore, our results suggest that the observed exponential decay in $E(t)$ is not caused by the redistribution of intracellular water within the cytoplasm under compression, which typically takes a longer time of the order of 0.1-0.5 s [39]. In fact, the speed-dependent $F(t) / F_{0}$ can be calculated using a continuous piecewise linear loading function $\delta(t)$, as shown in the inset of Fig. 3(a) (see Appendix A 2 for more details). The calculated $F(t) / F_{0}$ (green dashed lines) agrees well with the measurements.

\section{Characteristics of power-law decay at long times}

When $t>t_{c}$, the measured $E(t)$ moves to the power-law decay regime covering a three-decade time span from $10 \mathrm{~ms}$ to $10 \mathrm{~s}$. The obtained power-law exponent $\alpha$ is in the range of $0.16-0.41$, depending on the type of cells (see Table I). The obtained values of $\alpha$ fall in the previously reported range of $0.1-0.5$ for living cells $[4,44,45]$. Power-law rheology with
$0 \leqslant \alpha \leqslant 1$ is used to characterize the slow reorganization of soft glassy networks [46]. A network behaves more like a solid if its value of $\alpha$ is close to zero, and behaves more like a fluid if its value of $\alpha$ is close to unity. The power-law relaxation for living cells was found to be associated with the F-actin network in the cytoplasm $[4,20,47]$. To further verify that the observed power-law relaxation is indeed linked to the F-actin network, we examine how the measured $E(t)$ changes when the live MDCK II cells are under different drug treatments to alter the dynamics of their F-actin network. Our drug treatments include depletion of the cellular level of adenosine triphosphate (ATP) by sodium azide and 2-deoxy-d-glucose (dATP), disruption of the actin network by Latrunculin-A (LtnA) and inhibition of the myosin II activity by Blebbistatin (Bleb).

Among these drug treatments, disrupting the actin network by LtnA produces the largest effect. Both $E_{0}$ and $E_{2}$ decrease significantly [Figs. 4(b) and 4(f)], and $\alpha$ increases [Fig. 4(g)], indicating that the actin network becomes softer and more fluid-like. Myosin II motors are cross-linkers of the actin network and inhibiting their activity by Bleb also reduces the values of $E_{0}$ and $E_{2}$. Depleting the ATP level produces little effect on $E_{0}$ and $E_{2}$. Using immunostraining and confocal microscopy, we find that the actin network is disrupted significantly by LtnA. dATP and Bleb produce similar effects on the actin network, but not as severe as that by LtnA (see supplementary Fig. S1 [48]). Similar drug effects are also observed for HeLa cells (see supplementary Figs. S2 and S3 [48] and Table I).

Figure 4(h) shows a comparison of the relaxation modulus $E(t)$ of different cell types. The measured $F(t) / F_{0}$ for a red blood cell (erythrocyte) only reveals an exponential relaxation and does not have a long-time power-law relaxation like many other adherent cells. This is because erythrocyte is a special suspension cell that only has a thin sheet of spectrin and actin adjacent to the plasma membrane and does not have a three-dimensional (3D) actin network filling in the cell body. The absence of an actin network results in the unique cell shape and mechanical properties of erythrocyte. We also measure $F(t) / F_{0}$ for the MDCK II cells that are fixed by paraformaldehyde (PFA). After PFA fixation, the molecules in the cytoplasm are covalently cross-linked so that the 




(e)



(b)

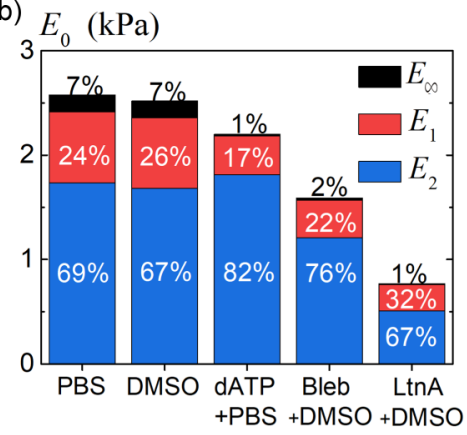

(f)

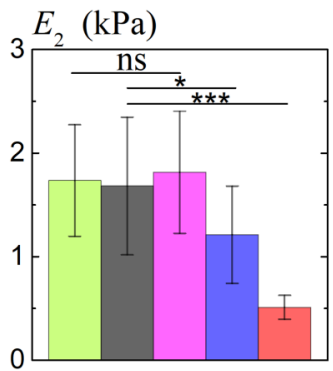

(c)

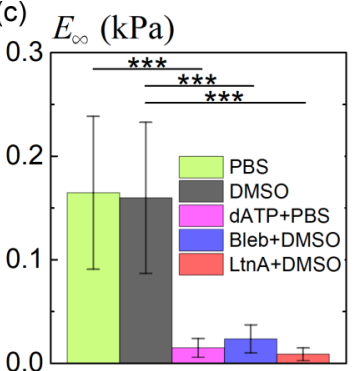

(g)

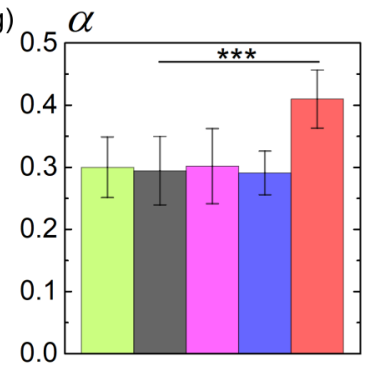

(d)

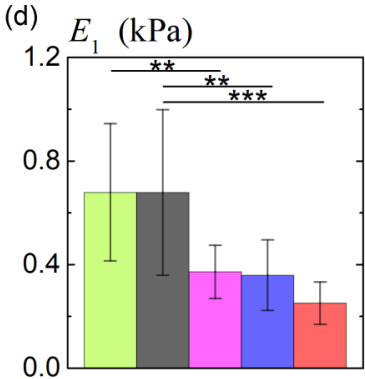

(h)



FIG. 4. Variations of relaxation modulus $E(t)$ under drug treatments. (a) Measured $F(t) / F_{0}$ for the MDCK II cells under different drug treatments: (i) ATP depletion (dATP, pink), (ii) inhibition of Myosin II activity (Bleb, blue), and disruption of actin network (LtnA, red). For comparison, control experiments are made in the PBS buffer and in the culture medium containing DMSO. The white solid lines show the fits of Eq. (1) to the data points. (b) Obtained initial modulus $E_{0}$ and its constituent components, $E_{\infty}, E_{1}$, and $E_{2}$, for the MDCK II cells under different drug treatments. (c)-(g) Obtained values of $E_{\infty}, E_{1}, \tau_{1}, E_{2}$, and $\alpha$ for the MDCK II cells under different drug treatments. The error bars indicate the standard deviations of the measurements. The inset of (c) shows the color codes used for (c) $-(\mathrm{g})$. The significant levels of the $t$-test are set as: not significant (ns) $p>0.05$, statistically significant $(*) 0.01 \leqslant p<0.05$, highly significant $(* *) 0.001 \leqslant p<0.01$, and extremely significant $(* * *) p<0.001$. (h) Measured $F(t) / F_{0}$ for three different cell types: (i) PFA-fixed MDCK II cell (blue curve), (ii) MDCK II cell (black curve), and (iii) erythrocyte (red curve). The white dashed lines indicate the fit of Eq. (1) to the data points. The fitting parameters are given in Table I.

entire cytoskeleton is frozen like a solid. Indeed, the measured $F(t) / F_{0}$ for the PFA-fixed cells is significantly slowed down and the power-law exponent $\alpha$ is changed from 0.30 (for live MDCK II cells) to 0.05 in line with the behavior of solids. Furthermore, the value of $E_{0}$ increases 5.7 times (see Table I).

\section{DISCUSSION}

The above results establish a unified description of the compressive modulus $E(t)$ of living cells, which is applicable to 10 different cell types studied. As shown in Eq. (2), $E(t)$ contains three relaxation modes with different timescales. We now provide some simple scaling analysis and order-of-magnitude estimate of the six parameters $\left(E_{1}, \tau_{1} ; E_{2}, \tau_{2}, \alpha ; E_{\infty}\right)$ involved in characterizing the three relaxation modes of $E(t)$. Our main aim here is to find the physical origin of each modulus component, so that these parameters could be used as effective indexes for "mechanical profiling" of living cells.

\section{A. Exponential decay at short times indicates a diffusive relaxation of confined proteins in cytoskeleton}

Various techniques, such as AFM compression, micromanipulator stretching and micropipette aspiration, have been used recently to study the stress relaxation of single cells $[34,35,39,45]$ and tissues [30,49]. Most of the studies found that the force relaxation $F(t)$ has an initial exponential-like decay with an apparent decay time $\tau_{1}^{\prime}$ in the range of $0.1-10 \mathrm{~s}$. This relaxation was attributed to solvent (water) redistribution within the meshwork of cytoskeleton, namely, poroelasticity of the actin network [39]. Because of the time dependence of the modulus $E(t)$, the obtained value of $\tau_{1}^{\prime}$ depends sensitively on the loading speed $v$, at which the cell deformation is applied. As shown in Fig. 3(b), the intrinsic decay time $\tau_{1}$ without much speed dependence is obtained only at the high speed limit, $v \gtrsim 100 \mu \mathrm{m} / \mathrm{s}$. Under this condition, the obtained values of $\tau_{1}$ for different cell types all range from 2 to $5 \mathrm{~ms}$ (see Table I), which is at least 20 times faster than those obtained in the previous experiments with a loading speed slower than $10 \mu \mathrm{m} / \mathrm{s}$.

This exponential decay can be explained by a diffusive relaxation of local concentration rearrangement of cytosolic proteins, which are partitioned into nanoscale domains by the deformed cytoskeleton under compression. The pore size $a$ of the cytoskeleton, therefore, becomes the largest length of concentration variations in the cytosol and their diffusive relaxation time is of the order of [50,51] $\tau_{1} \simeq a^{2} / D$, where $D$ is the diffusion coefficient of cytosolic proteins. With a typical value of $a \simeq 20 \mathrm{~nm}[52,53]$ and $D \simeq 0.15 \mu \mathrm{m}^{2} / \mathrm{s}$ [54], we find $\tau_{1} \simeq 2.7 \mathrm{~ms}$, which agrees with the typical values of $\tau_{1}$ obtained for different cell types (see Table I). In addition, we find the estimated value of the modulus component, $E_{1} \simeq k_{B} T / a^{3} \simeq 500 \mathrm{~Pa}$, agrees with the typical values of $E_{1}$ 
obtained for different cell types (see Table I). While details about the diffusion dynamics of cytosolic proteins across the cytoskeleton need to be further studied, the above numerical estimates indicate that the relevant length for the short-time exponential relaxation is the pore size $a$, rather than the indentation impact range $\Omega \simeq 3 \mu \mathrm{m}$. With these results, we conclude that the short-time exponential decay is determined primarily by the local concentration rearrangement of confined cytosolic proteins in the cytoskeleton.

\section{B. Power-law decay at long times reveals slow relaxation of deformed cytoskeletal network}

The long-time power-law decay in the measured $E(t)$ is caused primarily by the slow relaxation of deformed cytoskeletal network, which is made of cross-linked protein filaments, such as actin filaments (F-actin), microtubules and intermediate filaments. For eukaryotic cells, the mechanical response of their cytoskeleton is determined primarily by the F-actin network [2,5]. In the time domain, the power-law decay is described by $E_{2}\left(1+t / \tau_{2}\right)^{-\alpha}$, where the constant term of unity is introduced to separate the effect of the power-law relaxation from that of the short-time exponential relaxation. As shown in Fig. 1(c), the two relaxation modes are well separated by a crossover time $t_{c} \simeq 3 \tau_{2}(\sim 10 \mathrm{~ms})$, after which $E\left(t>t_{c}\right) \sim\left(t / \tau_{2}\right)^{-\alpha}$ for $t / \tau_{2} \gg 1$. Physically, $\tau_{2}$ represents the fastest relaxation associated with the smallest scale of the F-actin network, which is the mesh size $a$. This explains why the fitted value of $\tau_{2}$ is close to $\tau_{1}$.

The power-law rheology of living cells has been studied in the time domain by using the AFM and microplate techniques $[39,44,45]$, and in the frequency domain by using magnetic and optical tweezers $[4,22,34]$. In the frequency domain, the measured shear modulus takes the form, $G^{\prime}(\omega)=G_{0}\left(\omega / \omega_{0}\right)^{\alpha}$, where $G_{0}$ and $\omega_{0}$ are two scaling factors, which are often not independently determined as $\omega_{0}$ is often chosen to be $1 \mathrm{~Hz}$ for convenience. In this experiment, we are able to determine the absolute values of $E_{2}$ and $\tau_{2}$ with the combined measurements of $F(t)$ and $F(\delta)$ (see Table I). The power-law exponent $\alpha$ has been systematically evaluated in both the time and frequency domains for living cells and other soft glassy materials [55]. In our experiment, the obtained values of $\alpha$ range from 0.16 to 0.41 for 10 different cell types (see Table I), which fall in the previously reported range of $0.1-0.5$ [4,44,45]. With a typical binding energy $E_{b} \simeq 4 k_{B} T$ for actin-binding proteins (which are cross-linkers of the F-actin network) [56], we find that the estimated value of the actin network modulus, $E_{2} \simeq E_{b} / a^{3} \simeq$ $2 \mathrm{kPa}$, which agrees with the typical values of $E_{2}$ obtained for different cell types (see Table I).

\section{Origin of persistent modulus $E_{\infty}$}

For living cells, we find their relaxation modulus $E(t)$ does not decay to zero but reaches to an asymptotic value $E_{\infty}$, which is typically (2-10)\% of $E_{0}$ (see Table I). As shown in Fig. 4(c), the value of $E_{\infty}$ is reduced significantly under the three drug treatments, which reduce the activity of molecular motors and other active cellular processes. Furthermore, for the PFA-fixed cells, their $E_{\infty}$ is diminished to zero, even though their $E_{1}$ and $E_{2}$ increase drastically. These results prompt us to link $E_{\infty}$ to the active stress of the actin network generated by molecular motors.

Myosin II is a common type of molecular motor that constantly pulls the actin filaments and produces a persistent contractile stress in the cytoskeleton network [4,57]. When a living cell is under no external strain, its F-actin network is randomly orientated so that the contractile stress is approximately isotropic and acts like a negative pressure $-\sigma_{0}$. This contractile stress is balanced in part by the extensile stress generated by microtubules and, more importantly, by the external contacts of the adherent cell or its adhesion to the substrate [57,58]. When a living cell is vertically compressed by an external strain $\epsilon=\delta / H$, its F-actin network aligns primarily in the two horizontal directions. This "nematic ordering" increases the contraction in the two horizontal directions and reduces the contractile stress in the vertical direction $[59,60]$. In this case, we find that the scalar order parameter of the nematic phase is proportional to the external strain $\epsilon$ [61], and the corresponding modulus component $E_{\infty} \simeq 2 \sigma_{0} / 3$ is positive against compression (see Appendix B for more details).

When sliding on an actin filament with a step size $b \simeq$ $10 \mathrm{~nm}$, a single myosin II motor exerts a transient force $f_{m} \simeq 4 \mathrm{pN}$, or a mechanical energy of $f_{m} b \simeq 10 k_{B} T$, to the actin filament [62], which is approximately half of the free energy generated by a single ATP hydrolysis [57]. For a typical myosin II concentration of $n \simeq 4 \mu \mathrm{M}$ in nonmuscle cells [63], we find that the estimated value of the active modulus component $E_{\infty} \simeq n f_{m} b \simeq 100 \mathrm{~Pa}$, which agrees with the typical values of $E_{\infty}$ obtained for different cell types (see Table I). The corresponding persistent stress $E_{\infty}(\delta / H)$ obtained in our experiment also agrees with the previously reported values of the active stress for a reconstituted actin network $(\sim 10 \mathrm{~Pa})$ [64] and for the cell cortex $(\sim 100 \mathrm{~Pa})[9,65]$. Furthermore, our finding that the measured active stress $E_{\infty}(\delta / H)$ is about $(2-10) \%$ of the passive stress $\left(E_{1}+E_{2}\right)(\delta / H)$ for the living cells studied agrees with the calculations based on the hydrodynamic theory of active gels $[59,65]$.

Although the active modulus component $E_{\infty}$ is small compared with the other two passive components of $E(t)$, it plays an important role in cell migration and division, which are slow cellular processes with a typical time scale of several minutes $[9,11,57,58]$. During this period, the two passive components of $E(t)$ have decayed to zero. Because the production of active stress requires a continuing energy input from the ATP hydrolysis, depletion of the cellular ATP level results in a significant decrease in $E_{\infty}$, as shown in Fig. 4(c). Furthermore, because the actin filaments serve as the stress fibers for myosin motors, disruption of the actin network and its activity by LtnA and Bleb results in a significant decrease in $E_{\infty}$. With the above results, we conclude that the persistent modulus $E_{\infty}$ results mainly from the unbalanced active stress of deformed actin network under compression.

\section{Biological implications}

Unlike previous AFM indentation measurements, which only provide a single elastic modulus $E_{0}$ for a living cell at a particular loading speed, our combined stress relaxation and indentation force measurements clearly demonstrate that 
living cells are a special kind of viscoelastic materials with three relaxation modes at different timescales and can be characterized by a set of six parameters $\left(E_{1}, \tau_{1} ; E_{2}, \tau_{2}, \alpha ; E_{\infty}\right)$. With the accurate determination of the six viscoelastic parameters from the same cell, we are able to provide a coherent physical picture to quantify the interplay among the six viscoelastic parameters and their connections with specific biological parameters of the cell. This mechanical description of living cells is particularly important for the application of the AFM technique in analyzing the viscoelastic properties of healthy and diseased cells.

Living cells are often thought to have a thin stiff cortex overlying a much softer cytoplastic interior with a nucleus inside [10]. While the AFM measurements only sense the rheological rather than structural properties of the cell, our results shown in Figs. 1 and 2 nevertheless provide new insights into the cell cortex model. First, the cell cortex is not a pure elastic but viscoelastic sheet so that it has a relaxation modulus, which decays with time $t$. Second, although the cortical modulus is large compared with that of the cytoplastic interior, the mechanical contrast between the two components is not as large as was originally envisioned for a cortical shell with a liquid-like interior [66], in which case the measured indentation force would follow the relation $F(\delta) \sim \delta[30]$, instead of $F(\delta) \sim \delta^{3 / 2}$ as shown in Fig. 2(c). Third, because the three components of the cytoskeleton are interconnected and linked to the nucleoskeleton [67], it is difficult to distinguish the mechanical responses between the cytoskeletal network and the nucleus. Our model in Eq. (2) represents a coarse-grained description of the mechanical response of living cells as a three-dimensional continuum, even through the cell cortex and interior may have different contributions to the three components of the relaxation modulus $E(t)$. Further theoretical and experimental studies are needed to understand the spatial inhomogeneity of the cell.

With a proper calibration, the six parameters $\left(E_{1}, \tau_{1} ; E_{2}, \tau_{2}, \alpha ; E_{\infty}\right)$ as a matrix of mechanical indexes could be used to characterize the homeostasis of living cells in relation to their healthy and diseased states. To achieve this goal, one needs to control cell-to-cell variations that any quantitative measure of living cells will encounter. The experimental uncertainties can be reduced if the fitting parameters are obtained from the same batch of cells cultured under the same condition. For example, the red and blue curves in Fig. 1(d) show variations of the two stress relaxation curves obtained near the upper and lower bounds of the standard deviation. The first three rows in Table I give the fitting results from the three stress relaxation curves shown in Fig. 1(d). Except $E_{\infty}$ (which is very small compared with the other two components), the other fitting parameters obtained from the red and blue curves show less than $\pm 20 \%$ deviations from their mean values. These deviations are small compared with the effects caused by using different drug treatments (see Figs. 4(c)-4(g)) or cell types.

By systematically varying the control parameters for cell treatments, such as using different biochemical markers/inhibitors, cell line subtypes, or microenvironments including different substrate stiffnesses, one will be able to map out the landscape of the mechanical goals of living cells, identify the physical causes behind cellular desires, and provide a coarse-grained "mechanical profiling" for a given group of cells that complements molecular profiling, such as the gene expression and immune-histochemical expression profiling. For example, Table I reveals that the cancerous epithelial cells, such as HeLa cells, appear stiffer than normal epithelial cells, such as MDCK II and BEAS2B cells. In particular, the value of $E_{\infty}$ for HeLa cells is comparable to that for muscle cells and is significantly larger than that for MDCK II and BEAS2B cells. This result suggests that the contractile stress in cancer cells is enhanced, which agrees with the early finding [68] that cancer cells of various origins express a high level of myosin motors to drive cell protrusion formation and cell motility.

Our quantitative description of multiscale cell mechanics together with biological and biomedical methods will provide a reliable experimental framework that can be utilized to analyze the viscoelastic properties of healthy and diseased cells extracted from the same type of tissue and investigate in what ways a specific disease affects cell's physical properties. The systematic study of single cell mechanics will also pave the way forward for future studies of tissue mechanics. The present study is the first step toward this direction.

\section{MATERIALS AND METHODS}

\section{A. AFM setup and operation}

An AFM (MFP-3D, Asylum Research Inc.) was set up on an inverted microscope (IX71, Olympus) equipped with an EMCCD camera (Ixon3, Andor). The AFM used a colloidal probe for the stress relaxation and indentation force measurements. The cantilevers used in the experiment were tipless cantilevers (CSC12, MikroMasch) featuring a spring constant $0.6 \mathrm{~N} / \mathrm{m}$. The colloidal probe was assembled as described previously by adhering a glass sphere of diameter $d$ (in the range of 7-18 $\mu \mathrm{m}$ ) to the front end of the cantilever [69]. The surface of the colloidal probe was coated with a thin layer of PLL-g-PEG (SuSoS AG.), which effectively reduces the adhesion between the probe and living cell and also provides a high degree of resistance to protein adsorption in the cell culture medium (see the next section for more details). Prior to each force measurement, the spring constant of the colloidal probe is calibrated in situ using the thermal power spectral density method [69].

Live cells cultured on a coverslip are placed in a closed fluid chamber of the AFM. The AFM cantilever holder is mounted on the top of the fluid chamber, which is covered by a flexible rubber diaphragm to minimize liquid evaporation inside the chamber. An extra hole on the sidewall of the chamber is used for slow infusion of a humidified gas mixture of $95 \%$ air and 5\% $\mathrm{CO}_{2}$. All the force measurements for the living cells except Xenopus muscle cells were made at a temperature $\sim 37^{\circ} \mathrm{C}$, which is monitored and maintained by a local temperature control inside the fluid chamber. These experimental conditions ensured that the living cells under study were in a good physiological state. The inverted microscope was used to observe the location of the living cells and colloidal probe. By adjusting the scanning stage of the AFM, the colloidal probe is aligned on the top of a single cell and to face the central part of the cell body. After the alignment, AFM measurements were 
then performed on this cell. The force relaxation and indentation measurements were conducted at least twice on the same cell in different conditions (e.g., $v=1,10$, and $100 \mu \mathrm{m} / \mathrm{s}$ ) to make sure the data are repeatable.

\section{B. Surface treatment of AFM colloidal probe}

After assembly, the freshly made colloidal probe was plasma cleaned using a low-vacuum plasma cleaner (Harrick Plasma, PDC-32G) at the power of $40 \mathrm{~W}$ for $15 \mathrm{~min}$. The vacuum level of the cleaner was kept at about 600 milli-torr during the plasma cleaning. The colloidal probe was then coated with a thin layer of poly(L-lysine)-graft-poly(ethylene glycol) (PLL-g-PEG), which effectively reduces the cell adhesion to the probe and also provides a high degree of resistance to protein adsorption in the cell culture medium. The following is the coating procedure. First, an aqueous solution of $0.5 \mathrm{mg} / \mathrm{mL}$ PLL-g-PEG is made using the PLL-g-PEG powder (SuSoS AG.) and deionized water obtained from a Barnstead three-column e-pure system at the resistivity of 18 $\mathrm{M} \Omega \mathrm{cm}$. Then, the colloidal probe is placed in a disposable petri dish and a $100 \mu \mathrm{L}$ PLL-g-PEG solution is added to the petri dish to cover the whole body of the colloidal probe. The entire petri dish is sealed and the colloidal probe is kept in the PLL-g-PEG solution for 2 hours (or overnight) at room temperature. Finally, the PLL-g-PEG solution is removed, and the colloidal probe is rinsed with deionized water for three times. Incidentally, the coated PLL-g-PEG layer of a used colloidal probe can be readily removed by plasma cleaning. After plasma cleaning, the colloidal probe can be coated with PLL-g-PEG again and reused.

\section{Cell culture and drug treatments}

Ten different cell types ranging from epithelial, muscle and neuronal cells to blood and stem cells were used in this study. BEAS-2B cells, HeLa cells and HEK293T cells were cultured in Dulbecco's modified Eagle's medium (DMEM, Life Technologies); and MDCK II cells (Madin-Darby caninekidney cells) were cultured in Dulbecco's Modified Eagle Medium/Nutrient Mixture F-12 (DMEM/F-12, Invitrogen). Both media were supplemented with $10 \%$ fetal bovine serum (FBS) and penicillin/streptomycin (1×, Invitrogen). ReNcell VM human neural progenitor cells (Chemicon, SCC008) were cultured in ReNcell NSC Maintenance Medium (Millipore, SCM005) supplemented with $20 \mathrm{ng} / \mathrm{mL}$ basic fibroblast growth factor (bFGF) and $20 \mathrm{ng} / \mathrm{mL}$ of epidermal growth factor (EGF). Muscle cells were obtained from the dissection of myotomes of the fertilized Xenopus embryos as described previously [70], and raised in a culture medium composed of $88 \%$ Steinberg's solution, $10 \%$ L-15 medium (Leibovitz Co.), $1 \%$ fetal bovine serum and $1 \%$ gentamicin (50 mg/mL, Sigma-Aldrich) and maintained at $23^{\circ} \mathrm{C}$. Disassociated hippocampal neurons and glial cells were prepared from fetal mouse (E16.5) as described previously [71] and cultured in neurobasal medium (Invitrogen) supplemented with B-27 ( $1 \times$, Invitrogen), glutamine ( $2 \mathrm{mM}$, Invitrogen) and penicillin/streptomycin $(1 \times$, Invitrogen $)$. All cell cultures were maintained in a $95 \%$-air $/ 5 \%-\mathrm{CO}_{2}$ atmosphere at $37^{\circ} \mathrm{C}$ unless specified otherwise. Leukocytes and erythrocytes were prepared from $\sim 0.5 \mathrm{~mL}$ mice blood in an anticoagulanttreated tube and maintained in PBS on ice before use. To perform AFM measurements on these two nonadherent cells, the cells were seeded and allowed for attachment for $15 \mathrm{~min}$ on a poly-D-lysine $(0.5 \mathrm{mg} / \mathrm{mL}$ in PBS, Sigma) coated glass coverslip.

The following drug treatments were made to the MDCK II and HeLa cells to alter their activities and cytoskeletal network. (i) $3 \mathrm{mM}$ sodium azide and $30 \mathrm{mM}$ 2-deoxyglucose in PBS was treated to cells for $1 \mathrm{~h}$ to deplete the cellular level of adenosine triphosphate (dATP) and hence inhibit the motor activities. (ii) $20 \mu \mathrm{M}$ Blebbistatin (Bleb) dissolved in DMSO was added to the cell medium (DMSO:medium $=1: 1000$ ) for 30 mins to inhibit the activity of myosin II motors. (iii) $1 \mu \mathrm{M}$ Latrunculin-A (LtnA) dissolved in DMSO was added to cell medium for 30 mins to disrupt the F-actin network. After each drug treatment, the AFM force relaxation and force indentation measurements were made on the living cells in the same drug-treated medium.

The staining process was performed at room temperature. Briefly, cells were fixed with $4 \%$ paraformaldehyde in PBS (for $10 \mathrm{~min}$ ) and subsequently permeabilized with $0.2 \mathrm{M}$ $\mathrm{NH}_{4} \mathrm{CL} / \mathrm{PBS}$ containing $0.2 \%$ Triton $\mathrm{X}-100$ (for $10 \mathrm{~min}$ ), and then blocked with $4 \%$ bovine serum albumin (Sigma-Aldrich) in PBS (for $2 \mathrm{~h}$ ). To visualize the cell morphology and Factin network, F-actin and cell nucleus were stained with Phalloidin-TRITC (2 $\mu \mathrm{g} / \mathrm{mL}$; Sigma-Aldrich) and DAPI (1 $\mu \mathrm{g} / \mathrm{mL}$; Sigma-Aldrich) for $60 \mathrm{~min}$, respectively. Finally, the cell sample was washed with PBS (5 min each for three times) and mounted onto a microscope slide with Citifluor-AF-1 (Ted Pella), and then examined under a confocal microscope (LSM710, Zeiss).

\section{ACKNOWLEDGMENTS}

The authors wish to thank Q. Xu, X.-P. Xu, Y.-H. Wong, and J. Prost for illuminating discussions and S.-L. Chan, $\mathrm{W}$. $\mathrm{He}, \mathrm{H}$. $\mathrm{Wu}$, and $\mathrm{X}$. $\mathrm{Li}$ for providing live cell samples. This work was supported in part by NSFC under Grant No. 11972351 (D.G.), by the Hong Kong RGC under Grants No. 16306418 (D.G. and P.T.), No. 16302718 (P.T.), No. 16300421 (P.T.), No. 16102417 (P.H.), and No. 26302320 (R.Z.), and by MoST of Taiwan under the Grant No. 1102112-M-008-026-MY3 (P.Y.L.).

\section{APPENDIX A: THEORETICAL CALCULATIONS}

\section{Modified Hertz model with a time-dependent modulus $E(t)$}

Theoretical models that are used to extract the modulus $E$ from the force indentation measurement of $F(\delta)$ often assume that $E$ is a time-independent constant. For example, the widely used Hertz model [41] describes the contact mechanics between two elastic bodies with no surface adhesion as

$$
F(\delta)=\frac{4 R^{\frac{1}{2}} \delta^{\frac{3}{2}} E}{3\left(1-v^{2}\right)},
$$

where $F$ is the reacting force exerted on the spherical indenter of radius $R_{1}, E$ is the Young's modulus of the test material with an effective radius $R_{2}, R=1 /\left(1 / R_{1}+1 / R_{2}\right)$ is the effective radius at the contact, $v$ is the Poisson's ratio of the 
test material ( $v \simeq 0.5$ for living cells), and $\delta$ is the indentation depth of the test material under compression. Equation (A1) shows that $F(\delta)$ is a single-valued function of indentation $\delta$ and should not exhibit any speed dependence or hysteresis. This prediction does not agree with the experimental results for the living cells, as shown in Fig. 2. This is because the measured modulus $E(t)$ for living cells is not a constant but a time-dependent quantity, as shown in Fig. 1.

To account for the speed dependence and hysteresis of the measured $F(\delta)$, we modify the Hertz model by using the Ting's model [42] for a time-dependent modulus $E(t)$. According to the Ting's model, the constitutive equation for a linear viscoelastic material indented by a rigid axialsymmetric indenter with a continuous loading function $\delta(t)$ takes the following integral form:

$$
F(\delta, t)=\frac{4 R^{\frac{1}{2}}}{3\left(1-v^{2}\right)} \int_{0}^{t} E\left(t-t^{\prime}\right) \frac{\partial \delta^{\frac{3}{2}}}{\partial t^{\prime}} d t^{\prime} .
$$

For our AFM measurement with a constant loading speed $v$, we have $\delta(t)=v t$ for the advancing curve. With the timedependent modulus

$$
E(t)=E_{1} e^{-t / \tau_{1}}+E_{2}\left(1+t / \tau_{2}\right)^{-\alpha}+E_{\infty},
$$

we obtain

$$
F(\delta, t)=\frac{4 R^{\frac{1}{2}} \delta^{\frac{3}{2}} E_{0}}{3\left(1-v^{2}\right)} C^{*}(t),
$$

where

$$
\begin{aligned}
C^{*}(t)= & \frac{3}{2 E_{0} t^{3 / 2}} \int_{0}^{t} E\left(t-t^{\prime}\right) t^{\prime \frac{1}{2}} d t^{\prime} \\
\simeq & \frac{3}{2}\left(\frac{E_{1}}{E_{0}}\right)\left\{\frac{\tau_{1}}{t}-\frac{\sqrt{\pi}}{2}\left(\frac{\tau_{1}}{t}\right)^{\frac{3}{2}} e^{-t / \tau_{1}} \operatorname{Erfi}\left[\sqrt{\frac{t}{\tau_{1}}}\right]\right\} \\
& +\frac{3 \sqrt{\pi} \Gamma[1-\alpha]}{4 \Gamma\left[\frac{5}{2}-\alpha\right]}\left(\frac{E_{2}}{E_{0}}\right)\left(\frac{t}{\tau_{2}}\right)^{-\alpha}+\left(\frac{E_{\infty}}{E_{0}}\right) .
\end{aligned}
$$

In the above, Erfi[x] is the imaginary error function, which is a real function when $x$ is real, and $\Gamma[x]$ is the gamma function. Except for a correction factor $C^{*}(t)$, Eq. (A4) has a similar form as Eq. (A1).

\section{Calculation of the force relaxation $F(t)$ at different loading speeds $v$}

As shown in Fig. 3(a), the measured force relaxation $F(t)$ is slowed down when the loading speed $v$ of the AFM probe is reduced. This is because the cell deformation relaxes more for a slower indentation process. To quantitatively explain the effect of the speed-dependent $F(t)$, we calculate $F(\delta, t)$ using Eq. (A2) with the continuous loading function $\delta(t)$ given in Fig. 5 .

The final result is given by

$$
\begin{aligned}
F(\delta, t) & =\frac{4 R^{\frac{1}{2}} v^{\frac{3}{2}}}{3\left(1-v^{2}\right)}\left[\int_{0}^{t_{m}} \frac{3}{2} E\left(t-t^{\prime}\right) t^{\prime \frac{1}{2}} d t^{\prime}+0\right] \\
& \simeq \frac{4 R^{\frac{1}{2}}\left(v t_{m}\right)^{\frac{3}{2}} E_{0}}{3\left(1-v^{2}\right)}
\end{aligned}
$$

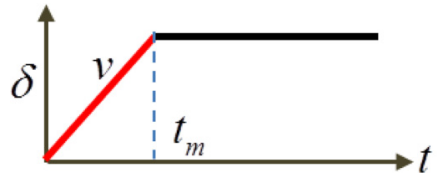

$$
\delta=\left\{\begin{array}{cc}
v t & 0<t<t_{m} \\
v t_{m} & t>t_{m}
\end{array}\right.
$$

FIG. 5. Continuous piecewise linear loading function $\delta(t)$ for the force relaxation measurement. The time origin $t=0$ is set when the AFM probe touches the upper surface of the cell. The red solid line indicates the linear increase of $\delta(t)$ at a constant loading speed $v$. At time $t=t_{m}$, the AFM probe stops and a constant value of $\delta$ is maintained (black solid line) for the measurement of the force relaxation $F(t)$ at $t>t_{m}$.

$$
\begin{aligned}
& \times\left[\frac{3 \tau_{1}}{4 t_{m}}\left(\frac{E_{1}}{E_{0}}\right)\left(2 e^{t_{m} / \tau_{1}}-\sqrt{\frac{\pi \tau_{1}}{t_{m}}} \operatorname{Erfi}\left[\sqrt{\frac{t_{m}}{\tau_{1}}}\right]\right) e^{-t / \tau_{1}}\right. \\
& +\left(\frac{E_{2}}{E_{0}}\right){ }_{2} F_{1}\left[\frac{3}{2}, \alpha ; \frac{5}{2} ; \frac{t_{m}}{t+\tau_{2}}\right]\left(1+\frac{t}{\tau_{2}}\right)^{-\alpha} \\
& \left.+\left(\frac{E_{\infty}}{E_{0}}\right)\right]
\end{aligned}
$$

where ${ }_{2} F_{1}[x]$ is the hypergeometric function. As shown in Fig. 3(a), the experimental results obtained at different loading speeds $v$ can be well described by Eq. (A6).

For a fast indentation $\left(t_{m} \rightarrow 0\right)$, we have $e^{t_{m} / \tau_{1}} \simeq$ $1+t_{m} / \tau_{1}$, Erfi $\left[\sqrt{t_{m} / \tau_{1}}\right] \simeq \frac{2}{\sqrt{\pi}}\left(\sqrt{t_{m} / \tau_{1}}+\frac{1}{3}\left(t_{m} / \tau_{1}\right)^{\frac{3}{2}}\right),{ }_{2} F_{1}\left[\frac{3}{2}\right.$, $\left.\alpha ; \frac{5}{2} ; \frac{t_{m}}{t+\tau_{2}}\right] \simeq 1$, and Eq. (A6) becomes

$$
F(t)=F_{0}\left[\frac{E_{1}}{E_{0}} e^{-t / \tau_{1}}+\frac{E_{2}}{E_{0}}\left(1+t / \tau_{2}\right)^{-\alpha}+\frac{E_{\infty}}{E_{0}}\right],
$$

where $F_{0}=\left(4 R^{\frac{1}{2}}\left(v t_{m}\right)^{\frac{3}{2}} E_{0}\right) /\left[3\left(1-v^{2}\right)\right]$. Equation (A7) recovers to Eq. (1) [or Eq. (A3)], once $F(t)$ is normalized by its maximum value $F_{0}$. Therefore, by measuring the normalized force relaxation $F(t) / F_{0}$ at a sufficiently fast loading speed $v(=100 \mu \mathrm{m} / \mathrm{s})$, one is able to determine the normalized relaxation modulus $E(t) / E_{0}$.

\section{Calculation of the retracting curve of $F(\delta, t)$}

To quantitatively explain the hysteresis effect between the approaching and retracting curves of the measured $F(\delta)$, we calculate the retracting curve of $F(\delta, t)$ using Eq. (A2) with the continuous loading function $\delta(t)$ given in Fig. 6. As mentioned above, the approaching curve $\left(0<t<t_{m}\right)$ of $F(\delta, t)$ is given by Eqs. (A4) and (A5). The calculated retracting curve

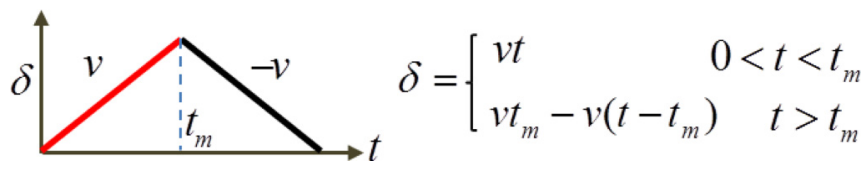

FIG. 6. Continuous piecewise linear loading function $\delta(t)$ for the approaching and retracting curves of force indentation measurements. The time origin $t=0$ is set when the AFM probe touches the upper surface of the cell. The red solid line indicates the linear increase of $\delta(t)$ at a constant loading speed $v$ during the approaching process. At time $t=t_{m}$, the AFM probe stops and retracts back from the cell surface at the same speed $v$ (black solid line). 
$\left(t>t_{m}\right)$ of $F(\delta, t)$ is given by

$$
\begin{aligned}
& F(\delta, t)=\frac{4 R^{\frac{1}{2}} v^{\frac{3}{2}}}{3\left(1-v^{2}\right)}\left[\int_{0}^{t_{m}} \frac{3}{2} E\left(t-t^{\prime}\right) t^{\prime \frac{1}{2}} d t^{\prime}-\int_{t_{m}}^{t} \frac{3}{2} E\left(t-t^{\prime}\right)\left(2 t_{m}-t^{\prime}\right)^{\frac{1}{2}} d t^{\prime}\right], \\
& =\frac{4 R^{\frac{1}{2}} v^{\frac{3}{2}}}{3\left(1-v^{2}\right)}\left[E_{\infty} t_{m}^{\frac{3}{2}}+\frac{3}{4} E_{1} \tau_{1} e^{-\frac{t}{\tau_{1}}}\left(2 \sqrt{t_{m}} e^{\frac{t_{m}}{\tau_{1}}}-\sqrt{\pi \tau_{1}} \operatorname{Erfi}\left[\sqrt{\frac{t_{m}}{\tau_{1}}}\right]\right)+E_{2} t_{m}^{\frac{3}{2}}\left(1+\frac{t}{\tau_{2}}\right)^{-\alpha}{ }_{2} F_{1}\left[\frac{3}{2}, \alpha ; \frac{5}{2} ; \frac{t_{m}}{t+\tau_{2}}\right]\right] \\
& -\frac{4 R^{\frac{1}{2}} v^{\frac{3}{2}}}{3\left(1-v^{2}\right)}\left\{E_{\infty}\left[t_{m}^{\frac{3}{2}}-\left(2 t_{m}-t\right)^{\frac{3}{2}}\right]\right. \\
& +\frac{3}{2} E_{1}\left[\tau_{1}\left(2 t_{m}-t\right)^{\frac{1}{2}}-\tau_{1}\left(t_{m}\right)^{\frac{1}{2}} e^{-\frac{t-t_{m}}{\tau_{1}}}+\frac{\sqrt{\pi}}{2} \tau_{1}{ }^{\frac{3}{2}} e^{\frac{2 t_{m}-t}{\tau_{1}}}\left(\operatorname{Erf}\left[\sqrt{\frac{t_{m}}{\tau_{1}}}\right]-\operatorname{Erf}\left[\sqrt{\frac{2 t_{m}-t}{\tau_{1}}}\right]\right)\right] \\
& \left.+E_{2}\left(\frac{t+\tau_{2}-2 t_{m}}{\tau_{2}}\right)^{-\alpha}\left(t_{m}{ }^{\frac{3}{2}}{ }_{2} F_{1}\left[\frac{3}{2}, \alpha ; \frac{5}{2} ;-\frac{t_{m}}{t+\tau_{2}-2 t_{m}}\right]-\left(2 t_{m}-t\right)^{\frac{3}{2}}{ }_{2} F_{1}\left[\frac{3}{2}, \alpha ; \frac{5}{2} ; \frac{t-2 t_{m}}{t+\tau_{2}-2 t_{m}}\right]\right)\right\} \\
& =\frac{4 R^{\frac{1}{2}}\left(v t_{m}\right)^{\frac{3}{2}} E_{0}}{3\left(1-v^{2}\right)}\left\{\left(\frac{E_{\infty}}{E_{0}}\right)\left(2-\frac{t}{t_{m}}\right)^{\frac{3}{2}}\right. \\
& -\frac{3}{2}\left(\frac{E_{1}}{E_{0}}\right)\left[\frac{\tau_{1}}{t_{m}}\left(2-\frac{\tau}{t_{m}}\right)^{\frac{1}{2}}-\frac{2 \tau_{1}}{t_{m}} e^{-\frac{t-t_{m}}{\tau_{1}}}+\frac{\sqrt{\pi}}{2}\left(\frac{\tau_{1}}{t_{m}}\right)^{\frac{3}{2}} e^{\frac{2 t_{m-t}-t}{\tau_{1}}}\left(\operatorname{Erf}\left[\sqrt{\frac{t_{m}}{\tau_{1}}}\right]-\operatorname{Erf}\left[\sqrt{\frac{2 t_{m}-t}{\tau_{1}}}\right]+e^{-\frac{2 t_{m}}{\tau_{1}}} \operatorname{Erfi}\left[\sqrt{\frac{t_{m}}{\tau_{1}}}\right]\right)\right] \\
& +\left(\frac{E_{2}}{E_{0}}\right)\left(1+\frac{t}{\tau_{2}}\right)^{-\alpha}{ }_{2} F_{1}\left[\frac{3}{2}, \alpha ; \frac{5}{2} ; \frac{t_{m}}{t+\tau_{2}}\right] \\
& \left.-\left(\frac{E_{2}}{E_{0}}\right)\left(\frac{t+\tau_{2}-2 t_{m}}{\tau_{2}}\right)^{-\alpha}\left[{ }_{2} F_{1}\left[\frac{3}{2}, \alpha ; \frac{5}{2} ;-\frac{t_{m}}{t+\tau_{2}-2 t_{m}}\right]-\left(2-\frac{t}{t_{m}}\right)^{\frac{3}{2}}{ }_{2} F_{1}\left[\frac{3}{2}, \alpha ; \frac{5}{2} ; \frac{t-2 t_{m}}{t+\tau_{2}-2 t_{m}}\right]\right]\right\} .
\end{aligned}
$$

As shown in Fig. 2(d), the calculated $F(\delta, t)$ in Eq. (A8) agrees well with the experimental results. Equations (A4) and (A8) thus quantitatively explain the differences between the approaching and retracting curves of the measured $F(\delta)$.

\section{APPENDIX B: CALCULATION OF ACTIVE MODULUS COMPONENT $\boldsymbol{E}_{\infty}$}

The orientational order of the actin filaments (F-actin) can be described by a symmetric and traceless tensor $\mathbf{Q}$, whose components are given by

$$
Q_{i j}=S\left(n_{i} n_{j}-\frac{1}{3} \delta_{i j}\right)
$$

where $S$ is the scalar nematic order parameter, $n_{i}$ is the $i$ thcomponent of a unit vector $\mathbf{n}$ representing the local average orientation of the F-actin, and $\delta_{i j}$ is the Kronecker delta function [72]. The active stress exerted by the myosin motors to the F-actin network can be written as

$$
\Pi_{i j}=\alpha\left(Q_{i j}+\frac{1}{3} \delta_{i j}\right),
$$

where $\alpha$ is the activity coefficient with a unit of stress $[59,60]$. It is known that a mechanical strain $\epsilon$ can induce a nematic order in the F-actin network. Specifically, it was found that $S \simeq \epsilon / 2$ [61]. A compressive strain in the vertical ( $z$ ) direction can induce a nematic order $S$ in the two horizontal directions by forcing the actin filaments rotate towards the horizontal plane. In terms of the $\mathbf{Q}$ representation, one has $\Delta Q_{x x}=$ $\Delta Q_{y y}=2 S / 3$. To preserve the tracelessness of the $\mathbf{Q}$ tensor, the change of the nematic order in the $z$-direction becomes $\Delta Q_{z z}=-4 S / 3 \simeq-2 \epsilon / 3$. In response to the decrease of the nematic order, a change of the normal stress in the actin network becomes $\Delta \Pi_{z z}=\alpha \Delta Q_{z z} \simeq-2 \alpha \epsilon / 3$. Therefore the active modulus component $E_{\infty}$ is given by $E_{\infty}=\left(\Delta \Pi_{z z}\right) / \epsilon \simeq$ $-2 \alpha / 3$. For most living cells, the active stress provided by the actin network is contractile, i.e., $\alpha<0$, and therefore one has $E_{\infty}>0$, in agreement with the experiments. In addition, the typical value of $\epsilon \simeq 0.2$ used in the experiment leads to a weak nematic order $S \simeq 0.1$, which is consistent with the literature value [61].
[1] V. Vogel and M. Sheetz, Local force and geometry sensing regulate cell functions, Nat. Rev. Mol. Cell Biol. 7, 265 (2006).

[2] P. A. Janmey and C. A. McCulloch, Cell mechanics: Integrating cell responses to mechanical stimuli, Ann. Rev. Biomed. Eng. 9, 1 (2007).
[3] K. E. Kasza et al., The cell as a material, Curr. Opin. Cell Biol. 19, 101 (2007).

[4] P. Kollmannsberger and B. Fabry, Linear and nonlinear rheology of living cells, Ann. Rev. Mater. Res. 41, 75 (2011). 
[5] E. Moeendarbary and A. R. Harris, Cell mechanics: principles, practices, and prospects, WIREs Systems Biology Med. 6, 371 (2014).

[6] A. F. Pegoraro, P. Janmey, and D. A. Weitz, Mechanical properties of the cytoskeleton and cells, Cold Spring Harb. Perspect. Biol. 9, a022038 (2017).

[7] D. E. Ingber, Tensegrity: The architectural basis of cellular mechanotransduction, Ann. Rev. Phys. 59, 575 (1997).

[8] P. P. Girard, E. A. Cavalcanti-Adam, R. Kemkemer, and J. P. Spatz, Cellular chemomechanics at interfaces: Sensing, integration and response, Soft Matter 3, 307 (2007).

[9] M. Stewart, J. Helenius, Y. Toyoda et al., Hydrostatic pressure and the actomyosin cortex drive mitotic cell rounding, Nature (London) 469, 226 (2011).

[10] E. Fischer-Friedrich, Y. Toyoda, C. J. Cattin et al., Rheology of the active cell cortex in mitosis, Biophys. J. 111, 589 (2016).

[11] M. Théry and M. Bornens, Get round and stiff for mitosis, HFSP J. 2, 65 (2008).

[12] I. Sokolov, S. Iyer, and C. D. Woodworth, Recovery of elasticity of aged human epithelial cells in vitro, Nanomed.: Nanotechnol. Biol. Med. 2, 31 (2006).

[13] S. E. Cross, Y. S. Jin, J. Rao, and J. K. Gimzewski, Nanomechanical analysis of cells from cancer patients, Nat. Nanotechnology 2, 780 (2007).

[14] M. Lekka, P. Laidler, and D. Gil, Elasticity of normal and cancerous human bladder cells studied by scanning force microscopy, Eur. Biophys. J. 28, 312 (1999).

[15] C. Rianna, M. Radmacher, and S. Kumar, Direct evidence that tumor cells soften when navigating confined spaces, Mol. Biol. Cell 31, 1726 (2020).

[16] R. M. Hochmuth, Micropipette aspiration of living cells, J. Biomechanics 33, 15 (2000).

[17] D. Sánchez et al., Noncontact measurement of the local mechanical properties of living cells using pressure applied via a pipette, Biophys. J. 95, 3017 (2008).

[18] O. Thoumine, A. Ott, O. Cardoso, and J. J. Meister, Microplates: a new tool for manipulation and mechanical perturbation of individual cells, J. Biochem. Biophys. Meth. 39, 47 (1999).

[19] I. Sokolov, M. E. Dokukin, and N. V. Guz, Method for quantitative measurements of the elastic modulus of biological cells in AFM indentation experiments, Methods 60, 202 (2013), Nanoimaging Methods for Biomedicine.

[20] F. Amblard, A. C. Maggs, B. Yurke, A. N. Pargellis, and S. Leibler, Subdiffusion and Anomalous Local Viscoelasticity in Actin Networks, Phys. Rev. Lett. 77, 4470 (1996).

[21] J. Dai and M. Sheetz, Mechanical properties of neuronal growth cone membranes studied by tether formation with laser optical tweezers, Biophys. J. 68, 988 (1995).

[22] B. D. Hoffman, G. Massiera, K. M. Van Citters, and J. C. Crocker, The consensus mechanics of cultured mammalian cells, Proc. Natl. Acad. Sci. USA 103, 10259 (2006).

[23] D. Weihs, T. G. Mason, and M. A. Teitell, Bio-microrheology: A frontier in microrheology, Biophys. J. 91, 4296 (2006).

[24] M. Nagayama, H. Haga, and K. Kawabata, Drastic change of local stiffness distribution correlating to cell migration in living fibroblasts, Cell Motility 50, 173 (2001).

[25] T. G. Kuznetsova, M. N. Starodubtseva, N. I. Yegorenkov, S. A. Chizhik, and R. I. Zhdanov, Atomic force microscopy probing of cell elasticity, Micron 38, 824 (2007).
[26] D. J. Müller and Y. F. Dufrêne, Atomic force microscopy: A nanoscopic window on the cell surface, Trends Cell Biol. 21, 461 (2011).

[27] M. Krieg et al., Atomic force microscopy-based mechanobiology, Nat. Rev. Phys. 1, 41 (2019).

[28] R. Matzke, K. Jacobson, and M. Radmacher, Direct, highresolution measurement of furrow stiffening during division of adherent cells, Nat. Cell Biol. 3, 607 (2001).

[29] D. Guan, E Charlaix, R. Z. Qi, and P. Tong, Noncontact Viscoelastic Imaging of Living Cells Using a Long-Needle Atomic Force Microscope with Dual-Frequency Modulation, Phys. Rev. Appl. 8, 044010 (2017).

[30] Y. Shen et al., Mechanical characterization of microengineered epithelial cysts by using atomic force microscopy, Biophys. J. 112, 398 (2017).

[31] C. J. Chan et al., Hydraulic control of mammalian embryo size and cell fate, Nature (London) 571, 112 (2019).

[32] Y. M. Efremov, T. Okajima, and A. Raman, Measuring viscoelasticity of soft biological samples using atomic force microscopy, Soft Matter 16, 64 (2020).

[33] E. Barthel, Adhesive elastic contacts: JKR and more, J. Phys. D: Appl. Phys. 41, 163001 (2008).

[34] E. Darling, S. Zauscher, and F. Guilak, Viscoelastic properties of zonal articular chondrocytes measured by atomic force microscopy, Osteoarthr. Cartil. 14, 571 (2006).

[35] A. N. Ketene, E. M. Schmelz, P. C. Roberts, and M. Agah, The effects of cancer progression on the viscoelasticity of ovarian cell cytoskeleton structures, Nanomed.: Nanotechnol. Biol. Med. 8, 93 (2012).

[36] Y. M. Efremov, W. H. Wang, S. D. Hardy, R. L. Geahlen, and A. Raman, Measuring nanoscale viscoelastic parameters of cells directly from AFM force-displacement curves, Sci. Rep. 7, 1541 (2017).

[37] P. D. Garcia, C. R. Guerrero, and R. Garcia, Nanorheology of living cells measured by AFM-based force-distance curves, Nanoscale 12, 9133 (2020).

[38] Y. Hu, E. P. Chan, J. J. Vlassak, and Z. Suo, Poroelastic relaxation indentation of thin layers of gels, J. Appl. Phys. 110, 086103 (2011).

[39] E. Moeendarbary, L. Valon, and M. Fritzsche, The cytoplasm of living cells behaves as a poroelastic material, Nat. Mat. 12, 253 (2013).

[40] M. Guo et al., Cell volume change through water efflux impacts cell stiffness and stem cell fate, Proc. Natl. Acad. Sci. USA 114, E8618 (2017).

[41] H. Hertz, On the contact of elastic solids, Z. Reine Angew. Math. 92, 156 (1881).

[42] T. C. T. Ting, The contact stresses between a rigid indenter and a viscoelastic half-space, J. Appl. Mech. 33, 845 (1966).

[43] M. Doi, Soft Matter Physics (Oxford University Press, 2013).

[44] M. Balland, N. Desprat, D. Icard, S. Fereol, A. Asnacios, J. Browaeys, S. Henon, and F. Gallet, Power laws in microrheology experiments on living cells: Comparative analysis and modeling, Phys. Rev. E 74, 021911 (2006).

[45] S. Hiratsuka et al., Power-law stress and creep relaxations of single cells measured by colloidal probe atomic force microscopy, Jpn. J. Appl. Phys. 48, 08JB17 (2009).

[46] P. Sollich, F. Lequeux, P. Hébraud, and M. E. Cates, Rheology of Soft Glassy Materials, Phys. Rev. Lett. 78, 2020 (1997). 
[47] L. Deng et al., Fast and slow dynamics of the cytoskeleton, Nat. Mater. 5, 636 (2006).

[48] See Supplemental Material at http://link.aps.org/supplemental/ 10.1103/PhysRevResearch.3.043166 for supplemental figures.

[49] N. Khalilgharibi, J. Fouchard, and N. Asadipour, Stress relaxation in epithelial monolayers is controlled by the actomyosin cortex, Nat. Phys. 15, 839 (2019).

[50] A. Kusumi, Y. Sako, and M. Yamamoto, Confined lateral diffusion of membrane receptors as studied by single particle tracking (nanovid microscopy) effects of calcium-induced differentiation in cultured epithelial cells, Biophys. J. 65, 2021 (1993).

[51] F. Daumas et al., Confined diffusion without fences of a gprotein-coupled receptor as revealed by single particle tracking, Biophys. J. 84, 356 (2003).

[52] K. Luby-Phelps, D. Taylor, and F. Lanni, Probing the structure of cytoplasm, J. Cell. Biol. 102, 2015 (1986).

[53] A. Derfus, W. Chan, and S. Bhatia, Intracellular delivery of quantum dots for live cell labeling and organelle tracking, Adv. Mater. 16, 961 (2004).

[54] H. Li et al., Mapping intracellular diffusion distribution using single quantum dot tracking: Compartmentalized diffusion defined by endoplasmic reticulum, J. Am. Chem. Soc. 137, 436 (2015).

[55] D. T. Chen, Q. Wen, P. A. Janmey, J. C. Crocker, and A. G. Yodh, Rheology of soft materials, Ann. Rev. Condens. Matter Phys. 1, 301 (2010).

[56] J. M. Ferrer et al., Measuring molecular rupture forces between single actin filaments and actin-binding proteins, Proc. Natl. Acad. Sci. USA 105, 9221 (2008).

[57] M. Sheetz and H. Yu, The Cell as a Machine, Cambridge Texts in Biomedical Engineering (Cambridge University Press, Cambridge, UK, 2018).

[58] U. S. Schwarz and S. A. Safran, Physics of adherent cells, Rev. Mod. Phys. 85, 1327 (2013).

[59] J. Prost, F. Jülicher, and J. F. Joanny, Active gel physics, Nat. Phys. 11, 111 (2015).
[60] M. E. Cates and E. Tjhung, Theories of binary fluid mixtures: from phase-separation kinetics to active emulsions, J. Fluid Mech. 836, 1 (2018).

[61] S. Majumdar, L. C. Foucard, A. J. Levine, and M. L. Gardel, Mechanical hysteresis in actin networks, Soft Matter 14, 2052 (2018).

[62] J. Finer, R. Simmons, and J. Spudich, Single myosin molecule mechanics: piconewton forces and nanometre steps, Nature (London) 368, 113 (1994).

[63] T. Luo et al., Understanding the cooperative interaction between myosin II and actin cross-linkers mediated by actin filaments during mechanosensation, Biophys. J. 102, 238 (2012).

[64] G. H. Koenderink et al., An active biopolymer network controlled by molecular motors, Proc. Natl. Acad. Sci. USA 106, 15192 (2009).

[65] J. Y. Tinevez et al., Role of cortical tension in bleb growth, Proc. Natl. Acad. Sci. USA 106, 18581 (2009).

[66] A. Yeung and E. Evans, Cortical shell-liquid core model for passive flow of liquid-like spherical cells into micropipets, Biophys. J. 56, 139 (1989).

[67] K. N. Dahl and A. Kalinowsk, Nucleoskeleton mechanics at a glance, J. Cell Sci. 124, 675 (2011).

[68] Y. R. Li and W. X. Yang, Myosins as fundamental components during tumorigenesis: diverse and indispensable, Oncotarget 7 , 46785 (2016)

[69] D. Guan et al., Direct measurement of optical force induced by near-field plasmonic cavity using dynamic mode AFM, Sci. Rep. 5, 16216 (2015).

[70] H. B. Peng, L. P. Baker, and Q. Chen, Tissue culture of Xenopus neurons and muscle cells as a model for studying synaptic induction, Meth. Cell Biol. 36, 511 (1991).

[71] M. Zeng et al., A binding site outside the canonical PDZ domain determines the specific interaction between shank and SAPAP and their function, Proc. Natl. Acad. Sci. USA 113, E3081 (2016).

[72] P. G. de Gennes and J. Prost, The Physics of Liquid Crystals (Clarendon Press, Oxford, 1993). 